\title{
Attitudes toward menopause in HIV-infected and at-risk women
}

\author{
Diana Hartel' \\ Yungtai Lo' \\ Carolyn Bauer ${ }^{2}$ \\ Nancy Budner' \\ Andrea A Howard' \\ Michelle Floris-Moore' \\ Julia H Arnsten ${ }^{1,2}$ \\ Nanette Santoro ${ }^{3}$ \\ Ellie E Schoenbaum ${ }^{1,2,3}$ \\ 'Departments of Epidemiology and \\ Population Health, ${ }^{2}$ Medicine, and \\ ${ }^{3}$ Obstetrics and Gynecology and \\ Women's Health, Montefiore Medical \\ Center and Albert Einstein College \\ of Medicine, Bronx, NY, USA
}

\begin{abstract}
Objective: To study attitudes toward menopause in women with or at risk of human immunodeficiency virus (HIV) aged 35 to 60 in New York City, NY, USA.

Design: Data were obtained at the baseline interview in a cohort study of menopause. Of 502 participating women, 92 were postmenopausal and 162 were perimenopausal.

Results: Overall, $37.5 \%$ of women had a relatively favorable attitude toward menopause. African Americans had a $72 \%$ greater odds of a positive attitude $(\mathrm{OR}=1.72,95 \%$ CI $1.16-2.57)$ than all other groups after adjusting for covariates. Hispanic women had the least favorable view of menopause. Experience of $>3$ menopausal symptoms and negative life events - being a witness to a murder, and the death of a child — were significantly associated with negative attitudes towards menopause ( $\mathrm{OR}=0.62,95 \%$ CI $0.42-0.93$ and $\mathrm{OR}=0.64,95 \%$ CI $0.43-0.93$, respectively). Depressive symptoms, street drug use, and having a domestic partner, which is significant in single variable analyses, did not remain independent predictors in multivariate results. HIV status, menopause status, and age at interview were not associated with menopause attitudes.

Conclusions: HIV-infected, drug-using, low-income women showed generally unfavorable attitudes towards menopause. High stress life events coupled with a high prevalence of depressive symptoms indicate this population has special needs marked by the menopause transition into older age.
\end{abstract}

Keywords: menopause, attitudes, HIV, street drug users

\section{Introduction}

The menopause marks a major biological transition in the lives of all women with potentially deep psychological and socioeconomic changes (Avis and McKinlay 1991). Attitudinal aspects of the menopause transition have been studied for several decades starting with studies by Neugarten and colleagues (1963). However, most studies of mid-life and menopause attitudes have systematically excluded women with serious illness, disability, or substance abuse.

As the human immunodeficiency virus (HIV) epidemic enters its third decade, a high percentage of women with HIV will also be entering menopause, their lives extended by improvements in antiretroviral therapies, ie, Highly Active Anti-Retroviral Therapy (HAART) (Palella et al 1998). Examination of the literature provides little insight into how women with HIV/acquired immunodeficiency syndrome (AIDS) or who use street drugs for long periods may experience the menopause transition.

We assessed attitudes towards menopause in an impoverished, urban population of midlife women with and at-risk for HIV infection. We hypothesized that HIV and street drug use would be associated with a less positive attitude toward menopause than for the HIV-uninfected women in our study who did not use drugs. We felt it equally plausible that menopause was just one more, relatively minor stressor among the many difficult circumstances among women with HIV as has been suggested by a number of investigators regarding HIV itself (Nyamathi 1992; Ward 1993). 


\section{Methods}

\section{Sample}

As previously described (Schoenbaum et al 2005), a total of 620 women participated in the "Natural History of Menopause in HIV-Infected Women" (Ms Study). Overall enrollment was guided to produce a study with balanced numbers of HIV-infected and uninfected women and with half of the women in the HIV-infected and uninfected groups having a history of heroin or cocaine use in the past five years. This study utilizes data gathered during the enrollment phase with ascertainment of menopause status with 12 months of amenorrhea postbaseline for those in the menopausal transition at time of interview.

\section{Definition of menopause, perimenopause, and menopausal symptoms}

Natural menopause was defined according to the World Health Organization (WHO 1996) as at least 12 consecutive months of amenorrhea not due to surgery or other obvious cause, such as extreme weight loss. Perimenopause was defined as absence of menses in at least 3 cycles but no more than 11 during the past 12 months. For this analysis we did not distinguish between early and late perimenopause due to small numbers of women in the perimenopause. Menopausal symptoms were classified into six groups, vasomotor, genitourinary, musculoskeletal, weight gain, palpitations, and insomnia and excluded psychological symptoms which were ubiquitous in the population (Miller et al 2005).

\section{Dependent variable}

A modified version of the Neugarten Attitudes Toward Menopause (ATM), a 35-item questionnaire, was administered as part of the baseline Ms interview. For each item on the ATM, women were asked to indicate if they agreed strongly, agreed somewhat, disagreed somewhat, or disagreed strongly or could not agree or disagree (don't know). Since the original Neugarten ATM was not designed for populations such as ours, a panel of 17 women familiar with the study population (primarily experienced research interviewers) selected a subset of ATM items. A $65 \%$ agreement was required by the panel in order to include an item. The items intended for middle-class, married women in the 1960s were dropped as inappropriate to a sample with an extensive history of street drug use and who are largely without partners. For example, we dropped: "After menopause, a woman gets more interested in activities outside the home." In the end we used 27 items (15 negative items, and 12 positive items) taken from the original ATM. Negative items were reverse coded for summation with positive items. Using this modified questionnaire, we condensed scores to a simple agree, don't know, or disagree code similar to scoring used for attitude items in the Study of Women Across the Nation (SWAN) (Sommer et al 1999). We examined variables in relation to average score and also to scores above 2, indicating more positive attitude, compared to those 2 and lower

\section{Exclusions}

Of the 620 women originally enrolled in Ms, women who had a hysterectomy and/or bi-lateral oophorectomy prior to entering the perimenopause $(n=22)$ were excluded. In addition, 24 women taking hormones for the symptoms of menopause during perimenopause within 6 months of interview were also excluded due to an inability to properly classify their status. Fifty-six women responding "don't know" more than $25 \%$ of the time on the ATM were dropped from analysis. Based on responses to questions on menopause symptoms, we found high nonresponse on the attitudes portion of the study instrument was likely to indicate lack of interest rather than simple lack of knowledge. A further 16 participants were excluded for incomplete or missing data. After the above exclusions, 502 women comprised the sample for this analysis.

\section{Statistical methods}

Bivariate analysis of baseline characteristics was performed using chi-square, Fishers' exact test, ANOVA, Spearman correlation, or Wilcoxon rank tests as appropriate. Multiple logistic regression models were used to identify factors independently associated with an average score above 2 compared with 2 or lower. Variables examined included age and age ${ }^{2}$, race/ethnicity, education, marital status, HIV status, CD4 + cell count, HAART treatment, drug and alcohol use, menopause status, menopause symptoms, parity, body mass index (BMI), negative life events, and depressive symptoms. Women were classed as having depressive symptoms if Center for Epidemiologic Studies Short Depression Scale (CES-D) scores $\geq 23$ as we have done in previous studies (Moore et al 1999; Schoenbaum et al 2005). Data were analyzed using Stata 9.0 (College Station, TX).

\section{Results}

Among the 502 women, 260 (51.8\%) were HIV-seropositive, and 92 women (18.3\%) were postmenopausal. For HIVpositive, median CD4 count was 455.5 cells $/ \mathrm{m}^{3}$ with an interquartile range (IQR) of 274-682. Sixty-four percent (168/261) were maintained on HAART at that time. 
The median age at interview was 43 (IQR: 40-46) with $27.3 \%$ reported using heroin and/or cocaine at the time of interview. The sample included 48.6\% African American, $40.2 \%$ Hispanic, 9\% non-Hispanic white, and 2.2\% "other", which included mixed race.

The overall average attitude toward menopause score (scores from 1 most negative to 3 most positive) was 1.93 with IQR of 1.77-2.10. Among 502 women, 37.5\% (188/502) had an average score $>2$ showing a relatively favorable attitude toward menopause. We found no differences in attitude toward menopause by HIV infection status: $37.3 \%$ of the $260 \mathrm{HIV}$-seropositive women and $37.6 \%$ of the 242 seronegative women had scores indicating a positive attitude toward menopause. There were no associations between CD4 count or use of HAART with menopause attitude among those with HIV. African American women showed the most positive attitudes toward menopause compared with all other race or ethnic groups (Table 1). Women reporting a current domestic partner (married or common-law) were less likely to have a positive attitude toward menopause than those with no partner $(32.1 \%$ vs. $41.3 \%, \mathrm{p}=0.035)$.

\section{Reproductive and medical history}

Menopause symptoms, but not menopause status, showed an inverse relationship with menopause attitude when women had more than 3 symptoms of any kind $(p=0.002)$ as shown in Table 1. Single menopause symptoms, such as hot flashes, were not associated with attitude score.

\section{Depressive symptoms and negative life events}

Depressive symptoms based on the CESD were related to a more negative menopause attitude score $(p=0.037)$. High rates of traumatic events were found among our participants: $42 \%$ abused as children and $36.1 \%$ molested as children, $45.8 \%$ raped and $59.4 \%$ assaulted as adults. In addition, $26.7 \%$ witnessed murder and $19.6 \%$ suffered a child's death. Witness to murder and death of a child showed a statistically significant difference in menopause attitude (Table 1).

\section{Drug and alcohol use}

For women reporting current use of heroin and/or cocaine in any form or route within 6 months of baseline interview, a somewhat more positive view of menopause was shown compared with nonusers ( $43.8 \%$ vs. $35.1 \%, \mathrm{p}=0.072)$. There was a similar result for problem drinking based on the CAGE screening instrument. Enrollment in methadone and cigarette smoking were not associated with attitudes toward menopause.

\section{Multivariate results}

African Americans showed 72\% greater odds of a positive attitude $\left(\mathrm{OR}_{\mathrm{adj}}=1.72,95 \% \mathrm{CI} 1.16-2.57\right)$ than all other race or ethnic groups while adjusting for the other variables in this model (Table 2). Experience of $>3$ menopausal symptoms and negative life events - being a witness to a murder, and the death of a child - were significantly associated with negative attitudes towards menopause $(\mathrm{OR}=0.62,95 \%$ CI $0.42-0.93$ and $\mathrm{OR}=0.64,95 \%$ CI 0.43-0.93, respectively). Depressive symptoms, street drug use, and having a domestic partner, which was significant in single variable analyses, did not remain independent predictors in multivariate results. HIV status, menopause status, and age at interview were not associated with menopause attitudes. Age, age ${ }^{2}$, and menopause status were examined in all models but dropped in the final model due to consistent lack of association.

\section{Discussion}

The most prominent finding in our study is the overall low level of women expressing a positive attitude $(37.5 \%$ positive, with 1.93 overall mean score) toward menopause in this impoverished population with a high prevalence of HIV. In contrast, the SWAN study (Sommer et al 1999) enrolled an ethnically diverse population and reported an overall mean "on the positive side of neutral." A comparable result in our population would be an overall average above 2.00; in fact, it was infrequent to observe average scores above two on most subcategories of variables examined in our study as shown in Table 1. Similar to SWAN, Wilbur and colleagues (1995) showed an overall nonnegative, largely neutral attitude toward menopause in a small, random and diverse sample of women. Since these studies do not use identical menopause attitude items, we do not compare actual overall mean results.

Our study population differs from others as it has the lowest educational levels ( $43 \%$ not completing high school), severe stressors including homelessness, street drug use and addiction, and adverse life events such as witness to murder, sexual abuse and rape. These factors probably colored overall attitudes toward their lives in general.

\section{Menopause status and symptoms}

Contrary to studies showing postmenopausal status associated with positive attitudes toward menopause (Neugarten et al 1963; Wilbur et al 1995; Sommer et al 1999), our postmenopausal women did not demonstrate more positive attitudes than peri- or premenopausal women. However, having more than 3 symptoms of menopause was associated with more negative menopause attitude in our data, suggesting that 
Table I Single variable associations with attitudes toward menopause score* in ( $=502)$ women enrolled in Menopause Study (Ms)

\begin{tabular}{|c|c|c|c|c|}
\hline & n (\%) & $\begin{array}{l}\text { Mean } \\
\text { score }\end{array}$ & $\begin{array}{l}\text { \% Positive } \\
\text { attitude }\end{array}$ & p-value* \\
\hline Age at baseline & & & & 0.757 \\
\hline $35-44$ & $253(50.4)$ & 1.93 & 39.1 & \\
\hline $45-49$ & $179(35.7)$ & 1.89 & 34.6 & \\
\hline $50-54$ & $49(9.8)$ & 1.98 & 36.7 & \\
\hline $55+$ & $21(4.2)$ & 2.00 & 42.9 & \\
\hline Race/Ethnicity & & & & 0.002 \\
\hline African American & $244(48.6)$ & 1.97 & 44.3 & \\
\hline Hispanic & $202(40.2)$ & 1.89 & 29.7 & \\
\hline White & $45(9.0)$ & 1.91 & 37.8 & \\
\hline Other & II (2.2) & 1.83 & 27.3 & \\
\hline Education (years) & & & & 0.498 \\
\hline$\leq \mathrm{II}$ & $218(43.4)$ & 1.90 & 35.8 & \\
\hline $12+$ & $284(56.6)$ & 1.94 & 38.7 & \\
\hline Marital status & & & & 0.035 \\
\hline Has partner & $209(4 \mid .6)$ & 1.89 & 32.1 & \\
\hline No partner & $293(58.4)$ & 1.95 & 41.3 & \\
\hline Menopause status & & & & 0.912 \\
\hline Menopause & $92(18.3)$ & 1.94 & 39.1 & \\
\hline Perimenopause & $162(32.3)$ & 1.93 & 36.4 & \\
\hline Premenopause & $248(49.4)$ & 1.91 & 37.5 & \\
\hline Menopause symptoms at baseline & & & & 0.002 \\
\hline $0-3$ symptoms & $311(62.0)$ & 1.95 & 42.8 & 0.332 \\
\hline$>3$ symptoms & $191(38.0)$ & 1.89 & 28.8 & \\
\hline \multicolumn{5}{|l|}{ Parity (number of livebirths) } \\
\hline None & $59(11.8)$ & 1.92 & 39.0 & \\
\hline $\mathrm{I}-2$ & $201(40.0)$ & 1.96 & 41.8 & \\
\hline $3-4$ & $170(33.9)$ & 1.90 & 34.1 & \\
\hline $5+$ & $72(14.3)$ & 1.90 & 31.9 & \\
\hline Body mass index (BMI) & & & & 0.604 \\
\hline$<18.5$ (underweight) & $7(1.4)$ & 2.01 & 57.1 & \\
\hline 18.5-24.9 (normal) & $126(25.1)$ & 1.93 & 38.9 & \\
\hline 25-29.9 (overweight) & 144 (28.7) & 1.94 & 38.9 & \\
\hline$\geq 3$ (obese) & $224(44.7)$ & 1.91 & 35.3 & \\
\hline HIV infection status & & & & 0.945 \\
\hline HIV-positive & $260(51.8)$ & 1.93 & 37.3 & \\
\hline HIV-negative & $242(48.2)$ & 1.92 & 37.6 & \\
\hline CESD Score & & & & 0.037 \\
\hline$\geq 23$ & $195(38.8)$ & 1.88 & 31.8 & \\
\hline$<23$ & 307 (6।.2) & 1.96 & 41.0 & \\
\hline Witness to murder & & & & 0.034 \\
\hline Yes & $134(26.7)$ & 1.88 & 30.0 & \\
\hline No & $368(73.3)$ & 1.94 & 40.2 & \\
\hline Abused as child & & & & 0.092 \\
\hline Yes & $211(42.0)$ & 1.90 & 33.2 & \\
\hline No & $291(58.0)$ & 1.96 & 40.6 & \\
\hline Molested as child & & & & 0.671 \\
\hline Yes & $181(36.1)$ & 1.92 & 38.7 & \\
\hline No & 321 (63.9) & 1.93 & 36.8 & \\
\hline Raped as adult & & & & 0.730 \\
\hline Yes & $230(45.8)$ & 1.92 & 38.3 & \\
\hline No & $272(54.2)$ & 1.93 & 36.8 & \\
\hline Attacked as adult & & & & 0.940 \\
\hline Yes & $298(59.4)$ & 1.93 & 37.6 & \\
\hline No & $204(40.6)$ & 1.92 & 37.3 & \\
\hline Death of a child & & & & 0.035 \\
\hline Yes & $105(20.9)$ & 1.88 & 28.6 & \\
\hline No & $397(79.1)$ & 1.94 & 39.8 & \\
\hline
\end{tabular}


Table I (Continued)

\begin{tabular}{llll}
\hline & $\mathbf{n}(\%)$ & $\begin{array}{l}\text { Mean } \\
\text { score }\end{array}$ & $\begin{array}{l}\text { \% Positive } \\
\text { attitude }\end{array}$ \\
\hline $\begin{array}{l}\text { Current drug user (cocaine/heroin) } \\
\text { User }\end{array}$ & $137(27.3)$ & 1.96 & 43.8 \\
$\quad$ Nonuser & $365(72.7)$ & 1.91 & 35.1 \\
Currently in methadone program & & & 0.072 \\
$\quad$ Yes & $175(34.9)$ & 1.90 & 33.7 \\
$\quad$ No & $327(65.1)$ & 1.94 & 39.5 \\
Problem drinking (any CAGE symptoms) & & & 0.206 \\
Yes & $269(53.6)$ & 1.94 & 40.9 \\
$\quad$ No & $233(46.4)$ & 1.90 & 33.5 \\
Current cigarette smoker & $339(67.5)$ & 1.92 & 38.4 \\
$\quad$ Smoker & $163(32.5)$ & 1.93 & 35.6 \\
Nonsmoker & & 0.087 \\
\hline
\end{tabular}

Note: *Chi-squared.

Abbreviations: CES-D, Center for Epidemiologic Studies Short Depression Scale.

symptoms may outweigh menopause status. The SWAN study found physical symptoms associated with attitudes, however these symptoms were not independent of menopause status in multivariate models. The role of symptoms in menopause can be complex in our population. Many of our study participants experience a wide range of conditions such as tuberculosis, HIV-related symptoms, and drug withdrawal that can overlap or closely resemble some symptoms of menopause. In our investigation of this issue, we found that women did not mis-attribute menopause symptoms to other conditions so much as they simply did not associate them with menopause (Johnson et al 2008). For example, 70\% attributed hot flashes to menopause and $29 \%$ attributed vaginal dryness to menopause. Our study population also differs from others in its high prevalence of early menopause at a median age of 47 (Schoenbaum et al 2005). The observed early menopause is supported in a subsample of the same Ms cohort members in which menopause symptoms are shown at younger ages than the general population (Miller et al 2005). Furthermore, analysis of reproductive hormone patterns in this population has shown that they are consistent with menopause status and support an earlier menopause onset (Santoro et al 2007). This study also confirmed that age and WHO menopause status classifications used in our study were significantly related to follicle stimulating hormone levels.

\section{Demographic results}

Consistent with other reports (Pham et al 1997; Sommer et al 1999), African American women had the most positive attitudes toward menopause: $44.3 \%$ positive compared with Hispanic women with $29.7 \%$ having a positive attitude. The literature shows less consistency for associations with educational attainment (McKinlay et al 1992), although the SWAN found higher education to be related to better attitude after controlling on age, menopause status, and other variables. With over $43 \%$ of women not completing high school and the fairly uniform low socioeconomic status of our participants, we found no such association.

Having a domestic partner-especially a supportive partner-has been reported as a factor in a positive attitude towards menopause (Avis et al 2004). A supportive network has similarly been shown to relate to a positive attitude toward the menopausal transition (Rice 2005). Whether married or common-law, having a domestic partner was related to less favorable attitudes toward menopause in our data but only in bivariate analyses.

\section{Stressful life events, depressive symptoms, and HIV infection status}

In our previous study (Miller et al 2005) we found that menopause symptoms were associated with depressive symptoms and negative life events; however, we did not

Table 2 Multiple logistic regression: Independent predictors of positive attitude toward menopause

\begin{tabular}{llll}
\hline Variable & $\begin{array}{l}\text { Adjusted } \\
\text { odds ratio }\end{array}$ & $\begin{array}{l}\text { 95\% confidence } \\
\text { interval }\end{array}$ & p-value \\
\hline Race/ethnicity & & & \\
$\quad \begin{array}{l}\text { African American } \\
\text { White }\end{array}$ & 1.718 & $1.156-2.566$ & 0.007 \\
$\begin{array}{l}\text { Hispanic } \\
\text { Death events* }\end{array}$ & 0.635 & $0.685-2.670$ & $\begin{array}{l}0.384 \\
\text { Reference } \\
\text { Menopause }\end{array}$ \\
\begin{tabular}{l} 
Symptoms $>3$ \\
\hline
\end{tabular} & 0.622 & $0.432-0.933$ & 0.021 \\
\hline
\end{tabular}

Note: *Death of a child or witness to murder. 
find depressive symptoms independently associated with menopause attitudes. Instead, in this study menopause symptoms and negative life events were related to attitude. The literature shows conflicting results on this issue with some reporting increases in depressive symptoms during the menopausal transition (Maartens et al 2002; Bromberger et al 2003; Freeman et al 2004), while others do not (Woods and Mitchell 1997; Rabkin et al 1997; Avis et al 2001).

We found that HIV infection status per se was not associated with menopause attitudes. In other studies, the psychological profile of impoverished women with HIV is more likely shaped by the milieu of poverty or drug problems than by HIV alone (Nyamathi 1992; Ward 1993). This appears to be the case for menopause as well.

\section{Conclusions}

For HIV-infected and drug-using women, the menopause transition is likely to be a very different experience than for women living in mainstream culture. Menopause is an important life event that can mark a change into a period of increasing health risk from cardiovascular disease, osteoporosis, and early death. It is notable that these women who once thought they would die of HIV in a short period are now facing menopause amidst the burdens of poverty, multiple high stress events, and age-related chronic disease conditions.

\section{Acknowledgments}

We are grateful for the dedicated work of the interviewers and support staff of this study. The Menopause Study (Ms) was funded through National Institute on Drug Abuse grant (RO1 DA13564) and a Center For AIDS Research grant from the National Institute on AIDS and Infectious Diseases Research (A1051519). The authors report no conflicts of interest.

\section{References}

Avis N, Assmann S, Kravigtz H, et al. 2004. Quality of life in diverse groups of midlife women: assessing the influence of menopause health status and psychosocial and demographic factors. Qual Life Res, 13:933-46.

Avis NE, Crawford S, Stellato R, et al. 2001. Longitudinal study of hormone levels and depression among women transitioning through menopause. Climacteric, 4:243-9.

Avis NE, McKinlay SM. 1991. A longitudinal analysis of women's attitudes toward the menopuase: results from the Massachusetts Women's Health Study. Maturitas, 13:65-79.
Bromberger J, Harlow S, Avis N, et al. 2004. Racial/ethnic differences in the prevalence of depressive symptoms among middle-aged women: the Study of Women's Health Across the Nation (SWAN). Am J Pub Health, 8:1378-85.

Bromberger JT, Assmann SF, Avis NE, et al. 2003. Persistent mood symptoms in a multiethnic community cohort of pre- and perimenopausal women. Am J Epidemiol, 158:347-56.

Freeman EW, Sammel MD, Liu L, et al. 2004. Hormones and menopausal status as predictors of depression in women in transition to menopause. Arch Gen Psychiatry, 61:62-70.

Johnson T, Cohen H, Howard A, et al. 2008. Attribution of menopause symptoms in HIV-infected or at-risk drug-using women. Menopause, 15:551-7.

Maartens LW, Knottnerus JA, Pop VJ. 2002. Menopausal transition and increased depressive symptomatology: a community based prospective study. Maturitas, 42:195-200.

McKinlay S, Brambilla D, Posner J. 1992. The normal menopause transition. Maturitas, 14:103-15.

Miller SA, Santoro N, Lo Y, et al. 2005. Menopause symptoms in HIVinfected and drug using women. Menopause, 2:348-56.

Moore J, Schuman P, Schoenbam E, et al. 1999. Severe adverse life events and depressive symptoms among women with, or at risk for, HIV infection in four cities in the United States of America. AIDS, 13:2459-68.

Neugarten BL, Wood V, Kraines RJ, et al. 1963. Women's attitudes toward the menopuase. Vita Humana, 6:140-51.

Nyamathi A. 1992. Comparative study of factors relating to HIV risk level of black homeless women. JAIDS, 5:222-8.

Palella FJ, Delaney KM, Moorman AC, et al. 1998. Declining morbidity and mortality among patients with advanced human immunodeficiency virus infection. $N$ Engl J Med, 338:853-60.

Pham KT, Grisso JA, Freeman EW. 1997. Ovarian aging and hormone replacement therapy: hormonal levels, symptoms, and attitudes of African-American and white women. J Gen Intern Med, 12:230-6.

Rabkin JG, Johnson J, Lin SH et al. 1997. Psychopathology in male and female HIV-positive and negative injecting drug users: longitudinal course over 3 years. AIDS, 11:507-15.

Rice V. 2005. Strategies and issues for managing menopause-related symptoms in diverse populations: ethnic and racial diversity. Am J Med, 118(Suppl 2):142-7.

Santoro N, Yungtai L, Moskaleva G, et al. 2007. Factors affecting reproductive hormones in HIV-infected, substance-using middle-aged women. Menopause, 15(5):1-7.

Schoenbaum EE, Hartel D, Lo Y, et al. 2005. HIV Infection, drug use, and onset of natural menopause. Clin Infect Dis, 41:1517-24.

Sommer B, Avis N, Meyer P, et al. 1999. Attitudes toward menopause and aging across ethnic/racial groups. Psychosom Med, 61:868-75.

Ward M. 1993. A different disease: HIV/AIDS and health care for women in poverty, Cult Med Psychiatry, 17:413-30.

Wilbur J, Miller A, Montgomery A. 1995. The influence of demographic characteristics, menopausal status, and symptoms on women's attitudes toward menopause. Women Health, 23:19-39.

Woods NF, Mitchell ES. 1997. Pathways to depressed mood for midlife women: observations from the Seattle Midlife Women's Health Study. Res Nurs Health, 20:119-29.

[WHO] World Health Organization Scientific Group. Research on the menopause in the 1990s. WHO Technical Services Department Series No. 866, Geneva, Switzerland: WHO, 1996. 